\title{
Correction to: Linear combinations of order statistics to estimate the position and scale parameters of the Generalized Pareto distribution
}

\section{Gianfausto Salvadori ${ }^{1,2}$}

Published online: 6 July 2021

(C) Springer-Verlag GmbH Germany, part of Springer Nature 2021

Correction to: Stochastic Environmental Research and Risk Assessment 16 (2002) 1-17

https://doi.org/10.1007/s00477-001-0080-2

In the original publication of the article, the author name spelling was incorrectly published.
The correct spelling of the author name is provided in this correction.

Publisher's Note Springer Nature remains neutral with regard to jurisdictional claims in published maps and institutional affiliations.

The original article can be found online at https:// doi.org/10.1007/s00477-001-0080-2.

\section{Gianfausto Salvadori}

gianfausto.salvadori@unisalento.it

1 Dipartimento Di Matematica e Fisica "Ennio De Giorgi", Università del Salento, Provinciale Lecce-Arnesano, P.O. Box 193, 73100 Lecce, Italy

2 Dipartimento di Ingegneria Civile ed Ambientale, Politecnico Di Milano, Milan, Italy 